\title{
STRUCTURED IDIOSYNCRASIES IN VOWEL SYSTEMS
}

\author{
Jean-Luc Schwartz (1), Lucie Ménard (2)
}

(1) GIPSA-lab, Université Grenoble Alpes, CNRS, France, jean-luc.schwartz@gipsa-lab.fr

(2) UQAM Montreal, QC H3C 3P8, Canada, menard.lucie@uqam.ca

\begin{abstract}
We display data on structured idiosyncrasies in vowel systems in five languages. Structures in idiosyncrasies are characterized here by patterns of correlated covariations between F1 values for two vowels in a system, for a set of speakers of the corresponding language. Correlations might reflect both local constraints between close vowels in front and back series, ensuring their distinctiveness, and global stability in height series possibly related to articulatory uniformity and articulatory reuse.
\end{abstract}

Keywords: articulatory uniformity, height degrees, phonology-phonetic interface

\section{INTRODUCTION}

It is well known that acoustic realizations of a given phonological category are highly variable. In healthy adults, variability is not random. It is partly driven by context - in relation to coarticulatory processes. Another crucial component is related to individual variability. Each speaker displays specificities called idiosyncrasies - that are characteristic of her/his way of realizing phonemic targets. Idiosyncrasies are partly due to physical traits related to sex, age, size and, individual anatomy. They also depend on socio-linguistic specificities that reflect the learning environment of the speaker. But there also exists a part that is not constrained by physical or socio-linguistic pressures, but rather emerges from the way the speaker has selected specific strategies for realizing the phonemes of her/his language ("free idiosyncrasies": see [1, 2]).

Idiosyncrasies might tell us a lot about the phonological/phonetic interface, since they reveal how a speaker selects articulatory gestures and/or acoustic cues that she/he finds adequate and representative of the corresponding phoneme. Hence, they provide us with a window on the cognitive phonetic representation of the phoneme category.

In our studies of the French vowel system, after a pilot study [3], we made an intriguing discovery [4], that it was the trend for speakers, despite individual variability, to maintain stable F1 values for a given height degree. That is, mid-high vowels /e ø o/ tended to occupy similar F1 values for a given speaker, even though this value could change largely from one speaker to another, and the same was true for mid-low vowels $/ \varepsilon œ \nu /$. In other words, there was a correlation between $\mathrm{F} 1$ values for different vowels in a height series. This discovery was in line with some pioneer observations such as Keating's proposal of "acoustic or articulatory uniformity" [5]. A few papers later reported such correlations between acoustic cues characterizing pairs of phonemes, for consonantal voicing [6] and place of articulation [7, 8].

These data suggest that idiosyncrasies have a global structure. This provides a type of individual appropriation of a general property of phonological systems, which Ohala introduced as the "maximal use of available features" (MUAF) principle [9]. MUAF refers to the fact that features in phonological systems tend to combine in a systematic way. For example, if a system contains a set of place of articulation (PoA) features for voiceless plosives, and if the system has a voiced vs. voiceless contrast for one PoA, the same contrast likely exists for all PoAs. This provides a structural correspondence at the phonological level of the structure in idiosyncrasies in consonantal voicing observed by Chodroff and Wilson [6].

In another example, vowel systems tend to have an equal number of height degrees for front unrounded and back rounded series [10], which is paralleled at the phonological level by the structure of idiosyncrasies in the production of height contrasts, which we observed [4].

The structure in idiosyncrasies sheds important light on the phonology-phonetic interface and the way it is represented in the speaker's brain. In the current research, we aimed to document and add to what is known about height vowel idiosyncrasies in various languages, explore whether these structured idiosyncrasies are specific to the French language or are more general, and determine how they are shaped in different languages.

\section{METHODS}

We examined multi-speaker databases of vowel formants gathered from the literature or obtained from colleagues who had access to certain databases. A systematic analysis process was applied to these data, directly inspired by the methodology used in the pioneer paper in the field [4]. 


\subsection{Vowel formant databases}

The two databases for French speakers were:

- The pioneer study, reported in [4], with ten repetitions of the ten French oral vowels /i y u e $\varnothing$ o $\varepsilon \propto \supset \mathrm{a} /$, pronounced in isolation by 12 French speakers (four 4-year-olds, four 8-yearolds and four adult male and female speakers).

- The same paradigm was used in [1] with ten pairs of French Canadian adult male siblings.

The second set of databases was obtained from Praat [11], from the "Tables" command:

- The data gathered by Peterson \& Barney for American English [12], with 50 male and 25 female speakers, and one repetition of each of

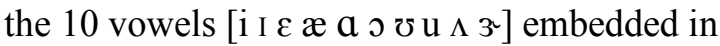
isolated monosyllabic words $/ \mathrm{h} \mathrm{V} \mathrm{d} /$.

- A database for Dutch by Pols et al. [13] provided the first three formants of 12 Dutch vowels [i I e $\varepsilon$ a a $\supset$ o u ø $\mathrm{Y}$ ] uttered by 50 male and 25 female speakers in an $/ \mathrm{h} \mathrm{V} \mathrm{d}$ / context.

We also gathered a database from the literature, and are grateful for several databases provided by colleagues:

- A more recent and complete database for American English by Hillenbrand et al. (1995) available on Internet [14] with one repetition of each of the 12 vowels [i I e $\varepsilon$ æ a $\rho$ o $v u \Lambda$ 3 ] by 45 adult males, 48 adult females, and 46 children in an $/ \mathrm{h} \mathrm{V} \mathrm{d} /$ context.

- A database for Spanish [15], provided by Julio González-Álvarez, with formant values for sustained phonation of each Spanish vowel [a e i o u] by 82 Spanish subjects ( 27 males, 55 females, aged 20 to 30 ).

- Databases for European and Peruvian Spanish [16], provided by Paul Boersma, with formant values for vowels $\left[\begin{array}{lllll}a & \mathrm{e} & \mathrm{i} & \mathrm{o} & \mathrm{u}\end{array}\right]$ in various consonantal contexts by 20 speakers ( 10 male, 10 female) of each variant.

- Databases for European and Brazilian Portuguese [17], provided by Paul Boersma, with formant values for vowels [a e i o u] in various consonantal contexts by 20 speakers (10 male, 10 female) of each variant.

- A database for Japanese [18], provided by Tatsuya Hirahara, with formant values for vowels [a e i o u] in isolation by 508 speakers (256 males, 252 females, aged 6 to 76).

\subsection{Analyses of structured idiosyncrasies}

We used the same method for normalizing data that was used by Ménard et al. [4]. That is, first we computed, for each speaker in each database, the minimum and maximum value of $\mathrm{F} 1 \mathrm{in} \mathrm{Hz}$, by taking the mean of $\mathrm{F} 1$ values for [i] and $[\mathrm{u}]$ for the minimum $\mathrm{F} 1^{\mathrm{min}}$, and the $\mathrm{F} 1$ value for [a] for the maximum $\mathrm{F} 1^{\text {max. }}$.

Then we defined, for each vowel for each speaker, a normalized F1 value (typically between 0 and 1, although this can be less than 0 or greater than 1 in rare cases) for each non-high or non-low vowel, using the formula:

$$
\mathrm{F} 1^{\text {Norm }}=\left(F 1-F 1^{\text {min }}\right) /\left(F 1^{\max }-F 1^{\text {min }}\right)
$$

Last, we computed regressions and Pearson correlation coefficients between pairs of vowels in a given language, and assessed the significance of correlation coefficients by Student's t tests.

\section{RESULTS}

\subsection{Confirming data for the height feature in French}

Regression patterns for mid-high and mid-low vowels in continental French (taken from [4]) are displayed in Figure 1. The pattern of correlation is quite clear and strong for mid-high vowels. It stays strong for the rounding contrast in mid-low vowels $[\varepsilon]$ vs. [œ], but it is much weaker for the contrast between the front unrounded and the back rounded contrast $[\varepsilon]$ vs. [ 0$]$. The pattern is amplified for Canadian French (Fig. 1, bottom, data from [1]). Indeed, the correlation completely vanishes for the $[\varepsilon]$ vs. [ $]$ contrast.
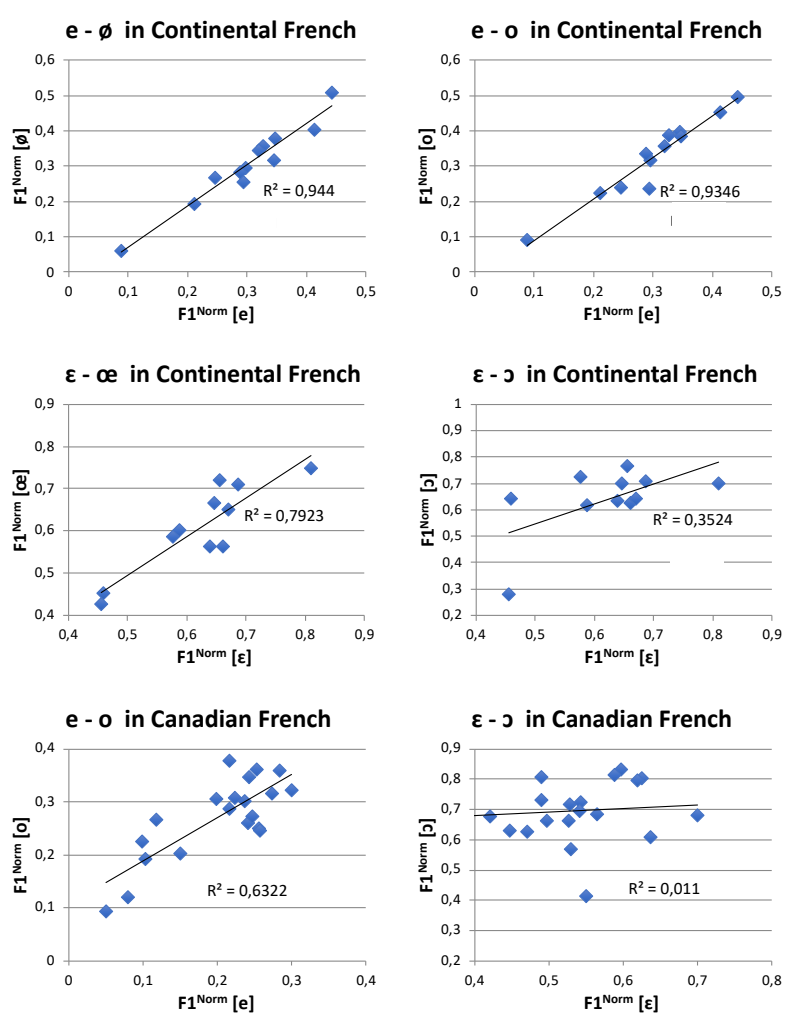

Figure 1: Patterns of correlation between normalized F1 values for the 12 speakers in [4] for mid-high and mid-low vowels in continental French, and for the 20 speakers in [1] for vowels in Canadian French. 


\subsection{Assessing the height feature in other languages}

We applied the same statistical methods for the other (non-French) languages in our databases. We found similar patterns of correlations for all languages, with large correlations for mid-high vowels, and smaller correlations for mid-low vowels. Figure 2 shows the correlation patterns, all highly significant $(\mathrm{p}<0.001)$, for a dense vowel system (American English, [14], with 12 vowels) and for a much less crowded system with 5 vowels (Spanish, [15], and Japanese, [18]).
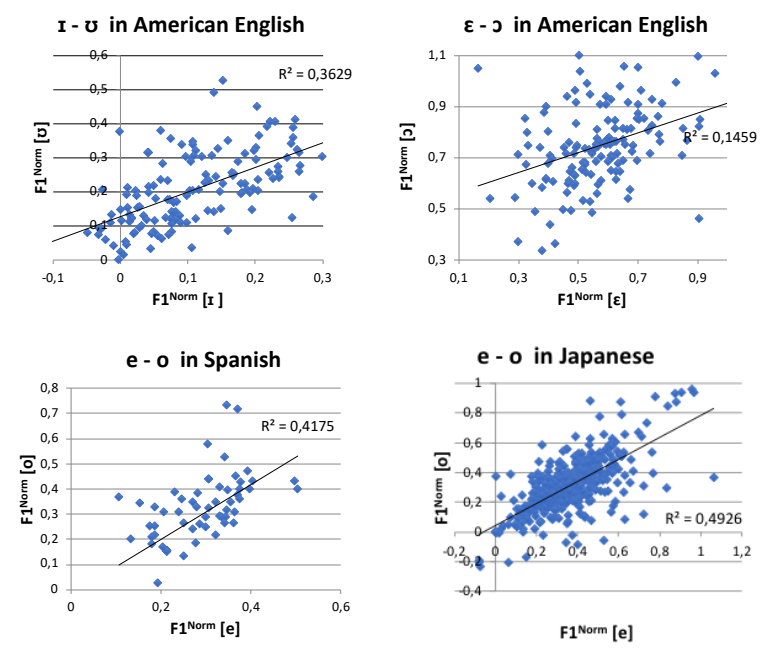

Figure 2: Patterns of correlation between normalized F1 values for the 139 speakers for American English in [14], the 82 speakers for Spanish in [15] and the 508 speakers in Japanese in [18].

\subsection{Correlations within front or back series}

While the previous analyses expanded on the initial discovery of structured idiosyncrasies within frontback and unrounded-rounded vowels sharing a height feature, we also wondered whether there could be correlations within front (unrounded or rounded) or back rounded series. It appears that such correlations do exist, as displayed in Figure 3. Interestingly, these correlations seem to exist more so for vowels that are close together in the vowel triangle (e.g. $[\mathrm{I}]$ and $[\mathrm{e}]$ in American English or $[\mathrm{e}]$ and $[\varepsilon]$ in Canadian French) but less so for distant vowels (e.g. [I] and $[\varepsilon]$ in American English. Note that the lack of a significant correlation for [o] and [0] in Canadian French is likely related to the ambiguous phonological status of the mid-low vowel / $/$ /. Indeed, the / $/ / \mathrm{vs}$. /a/ contrast is often neutralized, leading to the production of the low variant, far from the mid-high [o] and with F1 variations uncorrelated with those of the front midlow $[\varepsilon]$ (see Fig. 1)
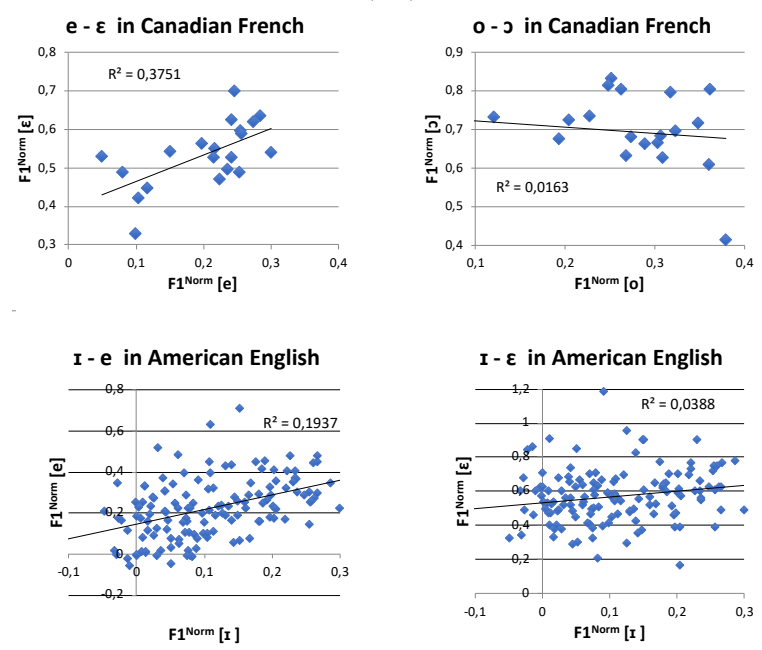

Figure 3: Patterns of correlation between normalized F1 values for pairs of vowels with front or back series, for Canadian French [1] and American English [14].

\section{DISCUSSION}

When we undertook this analysis of structured idiosyncrasies in vowel systems, we had no strong preconceived hypothesis. Surprisingly, 10 years after our observations of stable F1 values for height degrees in French, no replication or extension of these findings had been proposed in the literature. The present data reveal that idiosyncrasies in vowel systems display various kinds of structures, as shown in Figure 4 and Table 1. These structures seem to obey two different principles.

\subsection{Local interferences within front or back series}

The correlations between vowels within front (unrounded or rounded) or back series, displayed in Fig. 3, appear to operate mainly between close vowels. In Table 1, we report for each pair of vowels the distance in normalized F1 between the two members of the pair. The trend for local interactions is rather clear: for the four involved languages, correlations vanish when differences are above 0.35 . A likely interpretation is that these correlations are due to local acoustic dispersion constraints ensuring discriminability of two neighbor vowels. For example, if [I] has a relatively high $\mathrm{F} 1$ value in American English, the value for [e] must increase accordingly to ensure that they are not confused.

\subsection{Global structures within height degrees}

For the height series, the pattern differs. It has two major properties. First, it can operate at large distances, as in Spanish or Japanese where the correlation between values for [e] and [o] is strong, even though there is little risk of confusion in this five-vowel system. Here, local pressures for ensuring 
distinguishability are unlikely. In our earlier study [4] we proposed that the goal could be acoustic/auditory, speakers attempting to ensure stable F1 values. But the Figure 2 shows that correlations are not always associated with stable F1 values, since the data sometimes depart clearly from the first bisector with equal F1 values for the two members of the pair (for examples $\mathrm{F} 1^{\text {Norm }}$ values for [I] are systematically 0.1 lower than for $[\mho]$ in American English). Therefore, this is probably more in line with articulatory/geometric uniformity through articulatory reuse $[5,8,19]$, which is a regularizing pressure for exploiting the same articulatory component in various phoneme configurations. The question then is to determine what this articulatory component could be. Indeed, the stable F1 values for front [e] and back [o] in for example French or Spanish does not correspond to clear constraints in terms of for example tongue height of constriction area. Stability in jaw height could be a likely candidate, although this should be examined in future studies (see [4]).

The second major property in the present data concerns the large differences in height correlation between vowel pairs and between languages. While correlations were very strong in French, Spanish or Japanese, they were much weaker in American English or Dutch. Interestingly, the pattern of correlations in Canadian French also contributed to confirm the specific status of the lowest back rounded vowel as a low rather than a mid-low variant.

\section{CONCLUSION}

The search for structures in idiosyncrasies appears to be a rich paradigm, providing a very interesting window on vowel systems in human languages. They seem to display both local interferences within front and back series related to dispersion and discriminability, and global structures for height, possibly revealing articulatory reuse, but differing among height degrees and among languages.

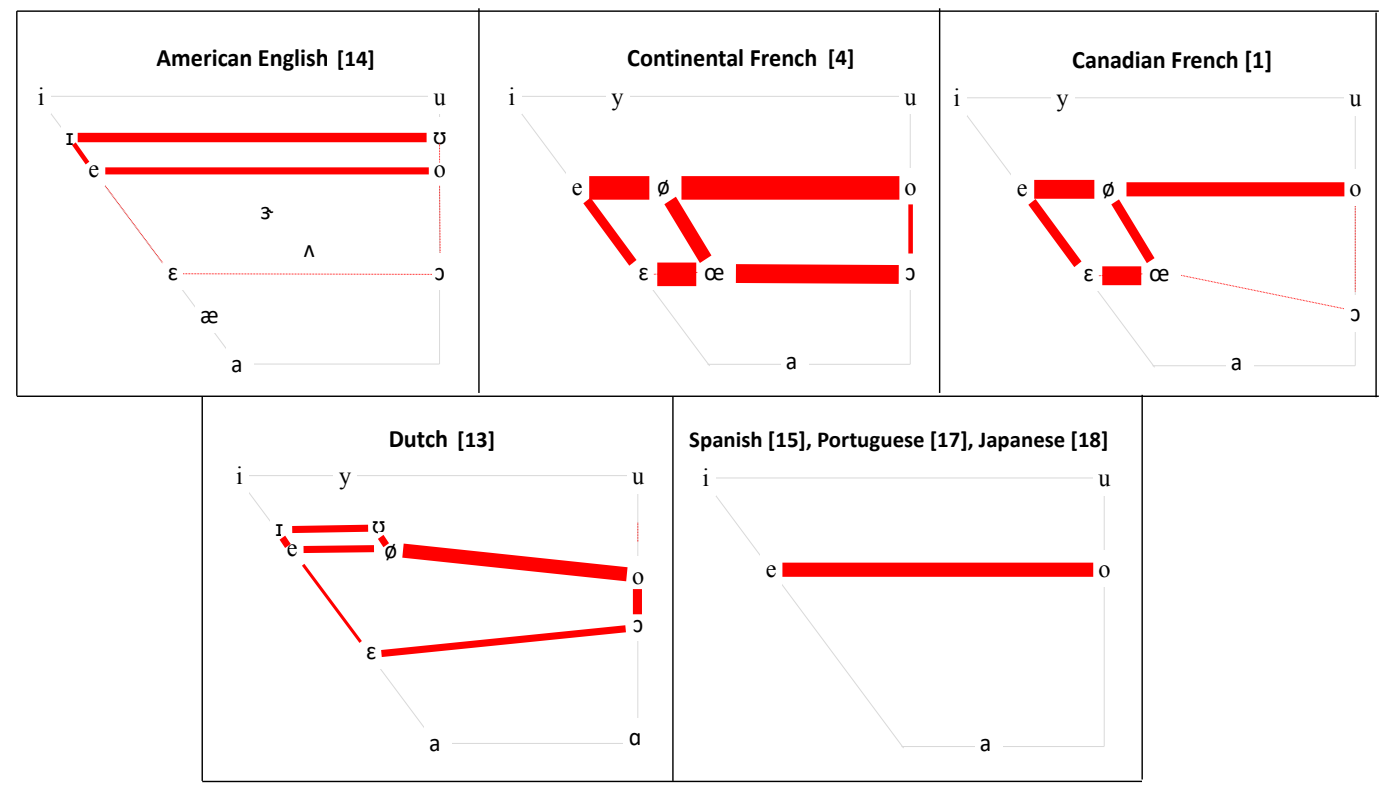

Figure 4: Graphical presentation of the covariations between pairs of vowels within and between front and back series for the five studied systems. The width of the line connecting two vowels in a given language is proportional to the corresponding correlation, shown in Table 1.

Table 1: Correlations (corr) between normalized F1 values for vowels in a given pair, for each tested language. For pairs within a given front or back series (in italics), deltaF1 provides the distance between mean $\mathrm{F} 1^{\text {Norm }}$ values in the pair.

\begin{tabular}{|c|c|c|c|c|c|c|c|c|c|c|c|c|c|}
\hline \multicolumn{3}{|c|}{ Continental French [4] } & \multicolumn{3}{|c|}{ Canadian French [1] } & \multicolumn{3}{|c|}{ American English [14] } & \multicolumn{2}{|c|}{ Dutch [13] } & \multicolumn{3}{|c|}{ Spanish [15] } \\
\hline & deltaF1 & corr & & deltaF1 & corr & & deltaF1 & corr & & deltaF1 & corr & & corr \\
\hline$e-\varepsilon$ & 0,31 & 0,30 & $e-\varepsilon$ & 0,33 & 0,38 & $I-e$ & 0,11 & 0,19 & $I-e$ & 0,03 & 0,26 & e-o & 0,42 \\
\hline$\varnothing-œ$ & 0,32 & 0,49 & $\varnothing-œ$ & 0,25 & 0,31 & $e-\varepsilon$ & 0,35 & 0,10 & $e-\varepsilon$ & 0,35 & 0,11 & & \\
\hline $\mathrm{e}-\varnothing$ & & 0,94 & e- $\varnothing$ & & 0,69 & $\mho-O$ & 0,07 & 0,12 & $0-0$ & 0,09 & 0,40 & & corr \\
\hline$\emptyset$-० & & 0,92 & $\varnothing-0$ & & 0,57 & $0-0$ & 0,47 & 0,04 & $\mathrm{I}-\mathrm{Y}$ & & 0,28 & e-o & 0,56 \\
\hline oe-כ & & 0,63 & oe-כ & & 0,04 & e-o & & 0,25 & e-o & & 0,15 & & corr \\
\hline$\varepsilon-\supset$ & & 0,35 & $\varepsilon-J$ & & 0,01 & $\varepsilon-כ$ & & 0,07 & $\varepsilon-J$ & & 0,28 & e-o & 0,49 \\
\hline
\end{tabular}




\section{Acknowledgements}

This work received funding from the ERC under the European Community's Seventh Framework Program (FP7/2007-2013 Grant Agreement no. 339152 - "Speech Unit(e)s"). We thank Julio González-Álvarez, Paul Boersma and Tatsuya Hirahara for having kindly provided us with their vowel databases for the present analyses. We thank Marlene Busko for copy-editing the paper.

\section{REFERENCES}

[1] Rapin L., Schwartz J.L., Ménard L. 2017. Are idiosyncrasies in vowel production free or learned? A study of French vowels in biological brothers. $J$. Acoust. Soc. Am. 141, 3582.

[2] Barnaud, M.L., Schwartz, J.., Bessière, P., Diard, J. in revision. Computer simulations of coupled idiosyncrasies in speech perception and speech production with COSMO, a perceptuo-motor Bayesian model of speech communication.

[3] Neagu, A., 1997. Analyse articulatoire du signal de parole: caractérisation des syllabes occlusive-voyelle en français. PhD dissertation, INPG, Grenoble France.

[4] Ménard, L., Schwartz, J.L., Aubin, J. 2008. Invariance and variability in the production of the height feature in French vowels. Speech Comm 50,14-28.

[5] Keating, P. 2003). Phonetic and other influences on voicing contrasts. Proc. ICPhS 15, 375-378

[6] Chodroff, E., Wilson, C. 2017. Structure in talkerspecific phonetic realization: Covariation of stop consonant VOT in American English. J. Phon. 61, 3047.

[7] Chodroff, E., Wilson, C. 2018. Predictability of stop consonant phonetics across talkers: Between-category and within-category dependencies among cues for place and voice. Linguistic Vanguard 4, S2 .

[8] Faytak, M.D. 2018. Articulatory uniformity through articulatory reuse: insights from an ultrasound study of Sūzhōu Chinese. PhD UC Berkely.

[9] Ohala, J.J., 1979. Moderator's introduction to symposium on phonetic universals in phonological systems and their explanation. Proc. ICPhS 3, 181185.

[10]Schwartz, J.L., Boë, L.J., Vallée, N., Abry, C. 1997. Major trends in vowel system inventories". J. Phon 25, 233-253.

[11]Boersma, P., Weenink, D. 2018. Praat: Doing phonetics by computer (Version 6.0.37). Retrieved from www.praat.org

[12]Peterson, G. E., Barney, H. L. 1952. Control methods used in a study of the vowels. J. Acoust. Soc. Am. 24, $175-184$.

[13]Pols, L.C.W. Tromp, H.R.C., Plomp R. 1973. Frequency analysis of Dutch vowels from 50 male speakers. J. Acoust. Soc. Am. 53, 1093-1101.

[14]Hillenbrand, J.M., Getty, L.A., Clark, M.J., Wheeler, K. 1995. Acoustic characteristics of American English vowels. J. Acoust. Soc. Am. 97, 3099-3111. https://homepages.wmich.edu/ hillenbr/voweldata.html

[15]González, J. 2004. Formant frequencies and body size of speaker: a weak relationship in adult humans. Journal of Phonetics, 32, 277-287.

[16]Chladkova, K., Escudero, P., Boersma, P. 2011. Context-specific acoustic differences between Peruvian and Iberian Spanish vowels. J. Acoust. Soc. Am. 130, 416-428.

[17] Escudero, P., Boersma, P, Schurt Rauber, A., Bion, R. 2008. A cross-dialect acoustic description of vowels: Brazilian and European Portuguese. J. Acoust. Soc. Am 126, 1379-93.

[18]Hirahara, T., Akahane-Yamada, R. 2004. Acoustic characteristics of Japanese vowels. Proc. 18th International Congress on Acoustics, Kyoto, IV-32873290.

[19]Lindblom, B. 1998. Systemic constraints and adaptive change in the formation of sound structure. In Approaches to the evolution of language: Social and cognitive bases (J.R. Hurford, M. Studdert-Kennedy, C. Knight Eds.) pp. 242-264. Cambridge: Cambridge University Press. 